\title{
Assistência de enfermagem primária a mulheres vítimas de violência perpetrada por
}

\section{parceiro íntimo}

\author{
Primary nursing care to women victims of violence perpetrated by intimate partner \\ Atención primaria de enfermería a mujeres víctimas de violencia por compañero intimo
}

Recebido: 28/10/2021 | Revisado: 07/11/2021 | Aceito: 10/11/2021 | Publicado: 14/11/2021

\author{
Hêmyle Thaynara Bezerra Corrêa \\ ORCID: https://orcid.org/0000-0003-1324-3178 \\ Centro Universitário Santo Agostinho, Brasil \\ E-mail: enfhemylethaynara@gmail.com \\ Gislena Martins Leal \\ ORCID: https://orcid.org/0000-0002-9826-4850 \\ Centro Universitário Santo Agostinho, Brasil \\ E-mail: lealgislena@gmail.com \\ Ana Livia Castelo Branco de Oliveira \\ ORCID: https://orcid.org/0000-0002-2634-0594 \\ Centro Universitário Santo Agostinho, Brasil \\ E-mail: analiviacbranco@unifsa.com
}

\begin{abstract}
Resumo
Introdução: A violência contra a mulher se caracteriza como um problema de saúde pública e de violação dos direitos humanos das mulheres podendo afetar negativamente a saúde física, mental, sexual e reprodutiva das mulheres e sendo praticada especialmente pelo parceiro íntimo. A assistência de enfermagem norteia o cuidar, facilitando o processo de atendimento mediado por intervenções direcionadas a saúde global da paciente. Objetivo: Identificar na literatura científica nacional e internacional evidencias sobre a assistência de enfermagem primária a mulheres vítimas de violência perpetrada por parceiro íntimo. Método: Revisão integrativa realizada nas bases de dados Latin American and Caribbean Health Science Literature via Biblioteca Virtual de Saúde e Web of Science, via Portal de Periódicos CAPES. Foram selecionados estudos originais, sem delimitações de linguagem ou temporalidade, que atendessem à questão de pesquisa. Os estudos foram submetidos à leitura de dois revisores independentes e após extração de dados, organizados em tabela sinóptica. Resultados: A maioria dos artigos foi buscado na base de dados Biblioteca virtual de saúde, a amostra de 13 artigos selecionados levantou questionamentos sobre a enfermagem aplicada a estratégias alternativas que contemplassem a saúde psíquica de mulheres que sofreram violência. Assim foram desenvolvidas intervenções de enfermagem que buscassem a identificação desse fenômeno e o correto manejo com fins de minimizar impactos físicos e psíquicos para as mulheres acometidas. Conclusão: Os estudos revelaram estratégias de intervenções de enfermagem às mulheres vítimas de violência especialmente física e psíquica e apontaram para a necessidade da capacitação das equipes de estratégia de saúde da família, enfatizando o cuidado integral e humanizado entre essas vítimas.
\end{abstract}

Palavras-chave: Enfermagem primária; Violência por parceiro íntimo; Violência contra a mulher; Saúde da mulher.

\begin{abstract}
Introduction: Violence against women is characterized as a public health matter, and as a violation of women's human rights, which can negatively affect the physical, mental, sexual and reproductive health of women and it is practiced especially by the intimate partner. Nursing assistance leads care facilitate the care process mediated by interventions directed to the patient's overall health. Objective: To identify evidence in national and international scientific literature on primary nursing care for women victims of violence perpetrated by an intimate partner. Method: Integrative review performed in the Latin American and Caribbean Health Science Literature databases in virtual health library and Web of Science, via CAPES Journal Portal. Original studies were selected, without language or temporal limitations, which met the research question. The studies were submitted to reading by two independent reviewers and after data extraction, organized in a synoptic table. Results: Most part of the articles were searched in the virtual health library database, the sample of 13 articles were selected in full about violence perpetrated against women by intimate partners, the studies raised questions about nursing applied to alternative strategies that contemplate the psychological health of women who have suffered violence. Therefore, nursing interventions were developed that sought to identify this phenomenon and its correct management in order to minimize physical and psychological impacts on affected women. Conclusion: The studies revealed nursing intervention strategies for women victims of especially physical and psychological violence and pointed to the need for training of family health strategy teams, emphasizing comprehensive and humanized care among these victims.
\end{abstract}

Keywords: Primary nursing; Intimate partner violence; Violence against women; Women's health. 


\begin{abstract}
Resumen
Introducción: La violencia contra la mujer se caracteriza como un problema de salud pública y una violación de los derechos humanos de la mujer. Esta puede afectar negativamente la salud física, mental, sexual y reproductiva de la mujer y es practicada especialmente por el compañero íntimo. El cuidado de enfermería orienta el cuidado, facilitando el proceso asistencial mediado por intervenciones dirigidas a la salud integral del paciente. Objetivo: Identificar evidencias en la literatura científica nacional e internacional sobre la atención primaria de enfermería a mujeres víctimas de violencia perpetrada por su compañero íntimo. Método: Revisión integrativa realizada en las bases de datos de Latin American and Caribbean Health Science Literature a través de la Biblioteca Virtual en Salud y Web of Science, a través del Portal de Revistas CAPES. Se seleccionaron estudios originales, sin limitaciones de lenguaje ni temporalidad, que cumplieran la pregunta de investigación. Los estudios fueron sometidos a lectura por dos revisores independientes y después de la extracción de datos, organizados en una tabla sinóptica. Resultados: La mayoría de los artículos fueron buscados en la base de datos de la Biblioteca Virtual en Salud, la muestra de 13 artículos seleccionados planteó interrogantes sobre enfermería aplicada a estrategias alternativas que abordan la salud psicológica de mujeres que han sufrido violencia. Así, se desarrollaron intervenciones de enfermería que buscaban identificar este fenómeno y su correcto manejo con el fin de minimizar los impactos físicos y psicológicos en las mujeres afectadas. Conclusión: Los estudios revelaron estrategias de intervención de enfermería para mujeres víctimas de violencia especialmente física y psicológica y señalaron la necesidad de formar equipos de estrategia de salud familiar, enfatizando la atención integral y humanizada de estas víctimas.
\end{abstract}

Palabras clave: Enfermería primaria; Violencia del compañero íntimo; Violencia contra la mujer; Salud de la mujer.

\title{
1. Introdução
}

A violência contra mulher, é definida como qualquer ação ou conduta que cause morte dano, sofrimento físico, sexual ou psicológico à mulher tanto em âmbito público como privado (Brasil, 1994). Fenômeno de definição antiga, porém recorre até hoje impactando a sociedade como um todo.

De acordo com o relatório da Organização Mundial da Saúde aproximadamente 35\% das mulheres sofreram violência física ou sexual por parceiro intimo entre os anos de 2012 e 2016 (Brasil, 2016).

Ainda existe muita dificuldade em compreender esse fenômeno, devido as conceituações nas diversas áreas do conhecimento, visto que essa problemática é discutida com intensidade pela psicologia, sociologia, saúde, segurança pública entre outros (Araújo et al., 2011). Contudo, sabe-se que a violência por parte de parceiro e a violência sexual são perpetradas principalmente por homens contra as mulheres, dados os seus determinantes culturais e sociais (Leite et al., 2019).

Nesta dinâmica, surge o instrumento auxiliador: o cuidado humanizado. Está diretamente ligado com o respeito a vida, essa prática deve estar presente em todos os cuidados especialmente a essas vítimas, tendo em vista que grande parte dos casos de violência perpetrada são detectados em âmbito de estratégia de saúde da família na atenção básica (Oliveira et al., 2020).

Baseado no exposto é importante a sensibilização dos profissionais de saúde que fazem parte da equipe de acolhimento, mais precisamente o enfermeiro, no cenário de atenção primária, que deve atuar com práticas humanizadoras. Esta atuação tem como roteiro, um protocolo de atendimento a mulheres vítimas de violência sexual, estabelecido pelo ministério da saúde com o objetivo de minimizar impactos da violência sexual (Brasil, 2012).

Logo, faz-se necessário conhecer o público, a pratica e os tipos de violência, e assim compreender as demandas para o combate deste fenômeno. Neste sentido, o presente estudo visa cobrir uma lacuna do conhecimento direcionando a assistência de enfermagem no cenário da violência sexual, visando orientar a sociedade sobre o manejo da problemática, especialmente nortear a pratica profissional, apresentando-lhe uma síntese de evidências nacionais e internacionais sobre o tema. Logo, foi objetivo deste estudo identificar na literatura cientifica nacional e internacional evidências sobra assistência de enfermagem primaria a mulheres vítimas de violência perpetrada por parceiro íntimo. 


\section{Metodologia}

O estudo é uma revisão integrativa, método que consiste na construção de uma análise ampla da literatura, contribuindo para discussões sobre métodos e resultados de pesquisas, assim como reflexões sobre a realização de futuros estudos (Mendes et al., 2008) aplicando a análise qualitativa que envolve uma abordagem interpretativa do mundo, o que significa que seus pesquisadores estudam as coisas em seus cenários naturais, tentando entender os fenômenos em termos dos significados que as pessoas a eles conferem (Denzin et al., 2006).

A proposta foi desenvolvida em seis etapas: 1. elaboração da pergunta norteadora; 2. definição das fontes de seleção dos estudos primários e dos critérios de inclusão e exclusão; 3. definição e extração dos dados a serem apresentados; 4. avaliação dos estudos incluídos; 5. análise crítica dos resultados; 6. apresentação da síntese das evidências encontradas (Whittemore; Knafl, 2005).

Para elaboração da pergunta norteadora, empregou-se a estratégia População - Interesse - Contexto (PICo), Quadro 1, na qual considerou-se: (P) mulheres vítimas de violência perpetrada por parceiro íntimo, (I) Assistência de enfermagem e (Co) Atenção primária em Saúde (Lockwood et al., 2017). Dessa forma, questionou-se: Quais as evidencias na literatura científica sobre a assistência de enfermagem primária a mulheres vítimas de violência perpetrada por parceiro íntimo?

Normalmente utilizada para elaboração de estudos não clínicos, esse acrônimo pode ser usado para desenvolvimento de questões de variadas, além de maximizar a recuperação de evidências nas bases de dados, foca o escopo da pesquisa e evita a realização de buscas desnecessárias (Lockwood et al., 2017).

Quadro 1 - Estratificação da pergunta de pesquisa: estratégia PICo e descritores controlados. Teresina, PI, Brasil, 2021.

\begin{tabular}{|c|c|c|}
\hline PICo & Componentes & Descritor DECs/ Mesh \\
\hline $\mathrm{P}$ & $\begin{array}{c}\text { Mulheres vítimas de violência } \\
\text { perpetrada }\end{array}$ & $\begin{array}{c}\text { Exposição a violência doméstica/ Exposure to violence } \\
\text { domestic }\end{array}$ \\
\hline $\mathrm{I}$ & Assistência de Enfermagem & $\begin{array}{c}\text { Enfermagem/Assistência de Enfermagem } \\
\text { Nurs } * / \text { Nursing care }\end{array}$ \\
\hline $\mathrm{Co}$ & Atenção primária & Atenção primária/ Primary Health Care \\
\hline
\end{tabular}

MeSH = vocabulário controlado da base Pubmed; DeCS = vocabulário controlado da base Lilacs. Fonte: Autores (2021).

Foram analisados estudos compilados nas seguintes bases de dados: Latin American and Caribbean Health Sciences Literature (Lilacs) via Biblioteca Virtual em Saúde (BVS) e Web of Science via coleção principal (Clarivate Analytics). O acesso às bases de dados ocorreu em agosto de 2021 a partir do portal de periódicos da Comunidade Acadêmica Federada (CAFe) e ao portal de periódicos da Coordenação de Aperfeiçoamento de Pessoal de Nível Superior (Capes).

Para seleção dos artigos que compuseram a amostra desta revisão, foram adotados os critérios de inclusão: estudos primários que atendessem a questão de pesquisa, disponíveis em qualquer idioma e sem delimitação temporal. Como critérios de exclusão, foram adotados: ser dissertação, tese, editorial ou outro estudo que utilizasse dados secundários.

Para realização da busca nas bases de dados, foram utilizados descritores controlados (termos estruturados hierarquicamente, utilizados na indexação nas bases) nos Descritores em Ciências da Saúde (DeCS) e seus correspondentes no Medical Subject Headings (MeSH). Inicialmente os descritores de cada conjunto da estratégia PICo foram combinados entre si com o conector booleano OR e, na sequência, cada conjunto foi combinado com o conector AND. A estratégia utilizada contou os descritores (“Atenção primária à saúde” OR "Enfermagem de atenção primária”) AND "Violência contra a Mulher".

A busca dos estudos, triagem e extração dos dados foram realizadas por duas pesquisadoras, que padronizaram a estratégia em cada base e a executaram de forma independente, com posterior comparação dos resultados encontrados.

A busca inicial resultou em 1.302 estudos na LILACS/BVS e WOF com 2446 estudos. Foram retirados os duplicados 
resultando em 989 estudos que foram submetidos à leitura de títulos e resumos. Foram excluídos 459 que estavam fora do tema e 390 estudos que eram a partir de dados secundários ou relato de experiência. Os 140 estudos restantes foram submetidos a leitura de texto completo, quando foram excluídos 128 por não atenderem à questão de pesquisa ou tratavam de forma elementar o fenômeno da violência. A amostra final foi representada por 13 estudos incluídos na síntese qualitativa dos dados.

\section{Resultados}

As variáveis coletadas versaram sobre as informações disponíveis nos estudos primários: título, autor, ano de publicação, base encontrada, nível de evidência, revista, método e objetivo. Para extração das informações contidas nos estudos primários, foi elaborado instrumento no Microsoft Office Word 2019, na forma de quadro sinóptico, para registro das variáveis apresentadas anteriormente. A descrição dos estudos que compuseram a amostra está disponível no Quadro 2.

Quadro 2 - Descrição dos estudos que compuseram a amostra. Teresina, PI, Brasil, 2021.

\begin{tabular}{|c|c|c|c|}
\hline TITULO & ANO & AUTOR & REVISTA \\
\hline $\begin{array}{l}\text { A violência conjugal expressa durante a gestação e puerpério: o } \\
\text { discurso de mulheres }\end{array}$ & 2019 & CAMPOS et al., & REME rev. min. enferm \\
\hline $\begin{array}{l}\text { Violência doméstica contra a mulher na percepção das equipes da } \\
\text { estratégia saúde da família }\end{array}$ & 2020 & OLIVEIRA et al., & $\begin{array}{l}\text { Rev. Pesqui. (Univ. Fed. } \\
\text { Estado Rio J., Online) }\end{array}$ \\
\hline $\begin{array}{l}\text { Violência por parceiro íntimo em abuso de álcool perpetrada } \\
\text { contra mulheres no climatério }\end{array}$ & 2019 & PIRES et al., & Rev. Enferm UFSM \\
\hline $\begin{array}{l}\text { Violência doméstica contra a mulher: visão de enfermeiros pós- } \\
\text { graduandos em obstetrícia no Piauí }\end{array}$ & 2019 & SANTOS et al., & Revista: J. nurs. Health. \\
\hline $\begin{array}{l}\text { Violência praticada por parceiros íntimos a mulheres com } \\
\text { depressão }\end{array}$ & 2020 & FRAZÃO et al., & REME rev. min. Enferm \\
\hline $\begin{array}{l}\text { Perfil da violência sexual contra mulheres atendidas no serviço de } \\
\text { apoio à mulher }\end{array}$ & 2017 & ALBUQUERQUE et al., & Rev. enferm. UFPE \\
\hline $\begin{array}{l}\text { Violência doméstica contra a mulher perpetrada por parceiro } \\
\text { íntimo: representações sociais de profissionais da atenção primária } \\
\text { à saúde }\end{array}$ & 2018 & SANTOS et, al & $\begin{array}{l}\text { Revista: Rev. pesqui. cuid } \\
\text { Fundam }\end{array}$ \\
\hline $\begin{array}{l}\text { Violência contra a mulher e sua associação com o perfil do } \\
\text { parceiro íntimo: estudo com usuárias da atenção primária }\end{array}$ & 2019 & LEITE et, al & Rev. bras. Epidemiol \\
\hline $\begin{array}{c}\text { Training healthcare providers to respond to intimate partner } \\
\text { violence against women }\end{array}$ & 2021 & KALRA et al., & $\begin{array}{c}\text { Cochrane Database Syst } \\
\text { Rev }\end{array}$ \\
\hline Women's primary care nursing in situations of gender violence & 2015 & VISENTIN et al., & Rev. Invest Educ Enferm \\
\hline $\begin{array}{l}\text { Psychic imprisonment through a psychoanalytic view: a married } \\
\text { aggressor case study }\end{array}$ & 2017 & STENZEL et al., & Rev. Agora (Rio J.) \\
\hline $\begin{array}{c}\text { Mental health effects of domestic violence against women in } \\
\text { Delhi: A community-based study }\end{array}$ & 2019 & SHARMA et al., & $\begin{array}{l}\text { Rev. J Family Med Prim } \\
\text { Care }\end{array}$ \\
\hline $\begin{array}{l}\text { Invisible conversations: subjects spoken but unheard in } \\
\text { gynecological visits }\end{array}$ & 2021 & MENEGHEL et al., & $\begin{array}{l}\text { Revista. C. \& Saúde } \\
\text { Coletiva }\end{array}$ \\
\hline
\end{tabular}

Fonte: Autores (2021).

\section{Discussão}

Os artigos compilados consistem no reconhecimento, combate e identificação de sinais de violência contra mulher, podendo ser ela física, sexual, moral, patrimonial e principalmente psíquica, aborda em seu contexto como deve ser o tratamento, abordagem e reconhecimento dessas vítimas a nível de atenção primária.

Com relação a categorização e seleção dos artigos são descritos elementos que possam ser usados pelos profissionais de saúde para identificação da violência, para que ocorra uma rápida ação proporcionando segurança e conforto à vítima 
(Oliveira et al., 2020).

É de suma importância a observação e preparo dos profissionais de saúde para o reconhecimento de expressões, identificação de lesões, ou recuamento, que são alguns dos sinais que podem ser apresentados por mulheres que sofreram ou sofrem com violência. Isto pelo viés da negação do ato em razão do medo do parceiro íntimo, trazendo assim inúmeras sequelas físicas e psicológicas a mulher (Albuquerque et al., 2017). Desta forma, os estudos destacaram os pontos de direcionamento da assistência de enfermagem por meio de tópicos relevantes sobre o tema.

Primeiramente, destaca-se a importância do reconhecimento das diversas formas de expressões de abusos experienciados por mulheres antes da gestação e podendo se manifestar até mesmo no puerpério, essas evidências poderão nortear o preparo profissional para o reconhecimento precoce do agravo no pré-natal (Campos et al., 2019).

Observou-se, que durante a gestação a violência perpetrada por parceiro íntimo torna ainda mais alarmante o impacto causado na saúde mental das mulheres (Oliveira et., al 2021) assim como na fase seguinte ao parto, no puerpério que é a fase de pós parto ao climatério que é considerado "o fim da vida reprodutiva" das mulheres existe a violência perpetrada por parceiro íntimo (Pires et al., 2019). Os autores relatam também, a violência contra mulheres na fase de climatério, tendo forte influência dos aspectos sociais e culturais, e do abuso de álcool.

No estudo de Albuquerque et al., (2017) foi identificado o perfil da violência sexual contra as mulheres atendidas num serviço de apoio à mulher, foi evidenciado o predomínio de mulheres vítimas de estupro com idades que variam de 20 à 49 anos, onde as agressões sexuais ocorreram à noite, em via pública, por agressor desconhecido que usou força física associada a ameaças para cometer o estupro por via vaginal contra as vítimas.

A violência pode ser cometida dentro ou fora de casa por algum membro da família, incluindo pessoas que passam a assumir função parental, ainda que sem laços de consanguinidade. $O$ agressor também possui relação de poder com a vítima especialmente em relação ao espaço físico onde a violência ocorre, mas também, às relações em que se constrói e efetua. (Cevs, 2017).

Quanto aos impactos para a vítima, pode-se mencionar a relação entre os conceitos de trauma, violência, abandono e pulsão por morte, vivencias ilustradas por estudo internacional (Stenzel et al., 2017). Em outra perspectiva, a violência por parceiro intimo pode resultar em impactos substanciais na saúde física e mental dos sobreviventes (Kalra et al., 2021). O estudioso reitera a importância do reconhecimento da violência perpetrada contribui para uma intervenção dos cuidados e resultados da saúde da mulher.

Outro estudo com mulheres que sofreram com violência doméstica mostrou o efeito significativo em saúde mental, trazendo traumas psíquicos, ocasionando pensamentos suicidas nessas vítimas, e frisa a importância de ser desenvolvidos intervenções de saúde pública que minimizem esse fenômeno (Sharma et al., 2019).

As questões culturais também possuem impacto nos contextos de violência e manutenção desta prática, como destacado no estudo de Santos et al., 2018, cuja a fala das participantes demonstrou ser a violência uma prática legitimada, aceita e tolerada, como algo imputado/inerente à mulher, onde se utilizou do ditado popular à banalização da violência conjugal contra mulheres. Em outra perspectiva a violência contra a mulher tem uma forte ligação com o fator sociocultural, onde foi considerado que a desigualdade de gênero implica na pratica da violência (Silva et al., 2020).

Embora tenham ocorridos avanços na política de saúde e proteção à mulher, a temática da violência por parceiro íntimo ainda é marcante na sociedade, deixando graves consequências às vítimas e suas famílias (Frazão et al., 2020).

Neste contexto, é primordial o desenvolvimento de ações primárias por profissionais de Enfermagem no sentido de identificar mulheres em situação de violência doméstica. Ainda que estes profissionais estejam permeados por sensação de impotência frente à gravidade e complexidade que envolve a violência (Visentin et al.,2015). Um dos momentos para esta detecção é a consulta para coleta de exame citopatológico, que pode ser oportuna para ouvir questões relacionadas à 
sexualidade e investigar a presença de violência contra a mulher (Meneghel et al., 2021).

Destaca-se em consenso com os demais estudos, a necessidade e a importância da capacitação dos profissionais da estratégia de saúde da família para a identificação e manejo adequado dos casos de violência doméstica contra a mulher, visando o cuidado integral (Oliveira et. al, 2020), pois existe a necessidade de planejamento, para acolhimento e coordenação do cuidado no atendimento às vítimas, que sentem medo e insegurança por conta da violência (Santos et al, 2019).

\section{Conclusão}

Identificou-se na literatura nacional e internacional artigos que destacavam a importância e a necessidade de capacitação teórico e pratica das equipes de estratégia de saúde da família a respeito da violência, tendo em vista que todos os dias mulheres de todas as idades independentemente do nível socioeconômico, tanto em âmbito público como privado sofreram ou sofrem algum tipo de agressão por parceiros ou terceiros.

Observou-se que por mais que tenha tido avanços atuais como a sanção da Lei Maria da Penha, em 2006 o número de mulheres violentadas tem sido um agravante, tornando-se um sério problema de saúde pública, econômica e social, essa é uma discussão necessária para a comunidade de discentes de enfermagem, para a comunidade científica em geral e para a sociedade, devido a recorrência da violência como prática ainda cotidiana nos cenários familiares, e considerados os prejuízos físicos e psíquicos trazidos pelo ato.

Assim, entende-se, a relevância de preparar os futuros profissionais de enfermagem para a assistência de modo que haja a identificação e manejo adequado dos casos de violência perpetrada contra a mulher, enfatizando o cuidado integral e humanizado entre essas vítimas, e para que sua realização possa servir de embasamento teórico, para construção de estudos futuros, tornando vasto o conhecimento e aprendizado.

\section{Agradecimentos}

Os autores agradecem a todos que direta ou indiretamente contribuíram para a construção e sucesso do artigo.

\section{Referências}

Albuquerque, A. L. de., \& Silva, W. C. da. (2017). Perfil da violência sexual contra mulheres atendidas no serviço de apoio à mulher. Revista de Enfermagem UFPE. 8(3)e:1032456.doi:10.5205/reuol.9302-81402-1-RV.1105sup201716

Campos, L., Gomes, N., Santana, J., Cruz, M., Gomes, N. \& Pedreira, L. (2019) Conjugal violence during pregnancy and puerperium: womens' discourse. Reme Revista Mineira de Enfermagem. 23(3) 10.5935/1415-2762.20190078.htpps://doi.org/10.5935/1415276220190078

Frazão M. C. L, Pimenta C. J. L. (2020). Violência praticada por parceiros íntimos a mulheres com depressão. Reme Revista Mineira de Enfermagem 24, e:1324. http://www.dx.doi.org/10.5935/1415-2762.20200061

Kalra, N., Hooker L., Reisenhofer, S., Di Tanna, G. L., \& García-Moreno C. (2021) Training healthcare providers to respond to intimate partner violence against women. Cochrane Database of Systematic Reviews, Issue 5. Art. No.: CD012423. 10.1002/14651858.CD012423

Leite, F. M. C. et al. (2019) Violência contra a mulher e sua associação com o perfil do parceiro íntimo: estudo com usuárias da atenção primária. Revista Brasileira de Epidemiologia, 22. https://doi.org/10.1590/1980-549720190056

Meneghel, S. N., Andrade, D. N. P., \& Hesler, L. Z. (2021). Conversas invisíveis: assuntos falados, mas não ouvidos em consultas ginecológicas. Ciência \& Saúde Coletiva.26(1). 275-284: <https://doi.org/10.1590/1413-81232020261.08012019>. https://doi.org/10.1590/1413-81232020261.0801201

Oliveira, G. L., Santos, N. de A., Machado, J. C., Pires, V. M. M. M., Morais, R. L. G. L., \& Rodrigues, V. P. (2021). Domestic violence against women according to the viewpoint of family health strategy teams / Violência doméstica contra a mulher na percepção das equipes da estratégia saúde da família. Revista De Pesquisa Cuidado é Fundamental Online, 12, 850-855. https://doi.org/10.9789/2175-5361.rpcfo.v12.7826

Oliveira, N. C. dos S., Gomes, N. L., Ferreira, T. R. de S. C., Santos, L. A., \& Franco, E. de P. (2020). Violência por parceiro íntimo durante a gravidez: um estudo baseado nos registros das capitais brasileiras. Research, Society and Development, 9(10), e599108342. https://doi.org/10.33448/rsd-v9i10.8342

Pires, Vilara \& Morais, Roberta \& Santos, Letícia \& Machado, Juliana \& Guedes, Caroline \& Rodrigues, Vanda. (2019). Violência por parceiro íntimo em abuso de álcool perpetrada contra mulheres no climatério. Revista de Enfermagem da UFSM. 9. e45. https://doi.org/10.5902/2179769234201 
Research, Society and Development, v. 10, n. 14, e591101422461, 2021

(CC BY 4.0) | ISSN 2525-3409 | DOI: http://dx.doi.org/10.33448/rsd-v10i14.22461

Santos, D. S., Magalhães, J. M., Côelho, M. C. V. S., Almeida, C. A. P. L, Viana, M. R. P. \& Carvalho, C. M. S. (2019). Violência doméstica contra a mulher: visão de enfermeiros pós-graduandos em obstetrícia no Piauí. J. nurs. Health. 9(3), e:199-310 DOI: HTTPS://DOI.ORG/10.15210/JONAH.V9I3.17198

Santos, W. J. dos, Oliveira, P. P. de, Viegas, S. M. da F., Ramos, T. M., Policarpo, A. G., \& Silveira, E. A. A. da. (2018). Domestic Violence Against Women Perpetrated by Intimate Partner: Professionals' Social Representations in Primary Health Care / Violência Doméstica Contra a Mulher Perpetrada por Parceiro Íntimo: Representações Sociais de Profissionais da Atenção Primária à Saúde. Revista De Pesquisa Cuidado é Fundamental Online, 10(3), 770-777. https://doi.org/10.9789/2175-5361.2018.v10i3.770-777

Sharma, K. K., Vatsa, M., Kalaivani, M., \& Bhardwaj, D. (2019). Mental health effects of domestic violence against women in Delhi: A community-based study. Journal of family medicine and primary care, 8(7), 2522-2527. https://doi.org/10.4103/jfmpc.jfmpc_427_19

Silva, A. F. C., Alves, C. G., Machado, G. D., Meine, I. R., Silva, R. M. da, \& Carlesso, J. P. P. (2020). Violência doméstica contra a mulher: contexto sociocultural e saúde mental da vítima. Research, Society and Development, 9(3), e35932363. https://doi.org/10.33448/rsd-v9i3.2363

Stenzel, G. Q. de L. \& Lisboa, C. S. de M, (2017) Aprisionamento psíquico sob uma perspectiva psicanalítica: estudo de caso de um agressor conjugal. Ágora: Estudos em Teoria Psicanalítica. 20, 625-633. <https://doi.org/10.1590/1809-44142017003003>. https://doi.org/10.1590/1809-44142017003003

Visentin, F. et al. (2015). Women's primary care nursing in situations of gender violence. Invest. educ. enferm 33(3), 556-564. https://doi.org/10.17533/ud ea.i ee.v33n $3 \mathrm{a} 20$ 